\title{
New oral anticoagulants in clinical practice - time to replace warfarin?
}

\author{
Indrakumar J \\ Journal of the Ceylon College of Physicians, 2017, 48, 41-45
}

Oral anticoagulation is the most fundamental treatment of thromboembolic disorders. Warfarin, has been in use since 1940's and in Sri Lanka at present is the only oral anticoagulant used in the prevention of stroke and venous thromboembolism. Regular coagulation monitoring and appropriate dose adjustments are important for safe and effective management. However, this could be a difficult task in some areas in our country where access to drugs and monitoring health institution is difficult and may need long distant travel.

Warfarin inhibits vitamin K-epoxide reductase and prevent the formation of vitamin $\mathrm{K}$ dependent clotting factors II, VII, IX, and X. It initially has a prothrombotic effect due to blocking proteins $\mathrm{C}$ and $\mathrm{S}$ before its actual antithrombotic effect begins ${ }^{1}$. It has a slow onset of action, usually started with a dose of 5-10 mg per day and the dose is adjusted according to the values of international normalized ratio(INR). Metabolism is mainly in the liver by cytochrome P-450 enzyme. Many factors such as comorbidities, genetic factors, and drugs can affect the function of this enzyme which can significantly induce or inhibit its metabolism leading to therapeutic problems. Food that are rich in vitamin $\mathrm{K}$ can inhibit the action of warfarin. Meta-analysis has shown that $44 \%$ of bleeding complications of warfarin is related to INR being above the therapeutic range and $48 \%$ of thrombo embolic episodes were related to INR being below the therapeutic ranges $^{2}$. Despite these drawbacks it has its advantages of availability, lower cost, once daily dosing and easily revisable properties.

An ideal anticoagulant would be expected to have a very good safety profile, rapid onset and offset of action, minimum drug and food interactions, predicable anticoagulant effect, wide therapeutic window, no need for monitoring, and have an effective antidote. The new oral anticoagulants (NOAC) demonstrates almost all the above characteristics and therefore increasingly becoming the preferred anticoagulant.

\section{New oral anticoagulants(NOAC)}

Previously warfarin was the main oral anticoagulant used and the only alternative had been parenteral

\footnotetext{
${ }^{1}$ Professor in Medicine, Department of Medicine, University of Sri Jayawardenepura, Sri Lanka.

E-mail: indrak2004@gmail.com
}

anticoagulants. NAOC's are now licensed for prevention of thromboembolism in non valvular atrial fibrillation and treatment and prevention of venous thromboembolism. These agents have almost all the advantages mentioned before, probably bring about a reduced overall cost to healthcare system ${ }^{3}$. However, lack of free availability, high cost of medications, lack of specific antidotes, and lack of long term safety data are still some of its drawbacks at present.

Currently there are 2 classes of NAOC's available. First is the direct thrombin inhibitors which act by inactivating fibrin bound thrombin ${ }^{4}$. Ximelagatran was the first approved drug in this class and was later withdrawn in 2006 due to high incidence of liver toxicity. Dabigatran is the next drug which do not cause liver toxicity and is currently approved for use. The second class of NAOC's rivaroxaban, apixiban, and edoxaban act by inhibiting the enzyme Factor $\mathrm{Xa}$ which is responsible for thrombin formation ${ }^{5}$. When compared to the action of warfarin which block the synthesis of factors II, VII, IX, and X, these agents block only a single step in coagulation pathway

\section{Venous thromboembolism (VTE)}

VTE has a high risk of recurrence and death. Anticoagulants are indicated for the management of both deep venous thrombosis (DVT) and pulmonary embolism (PE). All four NOAC's namely dabigatran ${ }^{6}$ rivaroxaban ${ }^{7}$, apixaban $^{8}$ and edoxaban ${ }^{9}$ are as effective as warfarin or lower molecular weight heparin. Clinically significant bleeding ${ }^{6,7,9}$ and major bleeding ${ }^{10}$ are significantly less than warfarin. Although it can be safely given as monotherapy in fixed doses without routine laboratory monitoring or dose adjustment, measurement of dug levels may be useful in certain clinical situations ${ }^{11}$.

Concomitant LMWH therapy for five days is required for dabigatran and edoxaban ${ }^{12,13}$ but not for rivaroxaban and apixaban ${ }^{14,15}$. Rivaroxaban and edoxaban can be given as once daily where as dabigatran and apixaban need to be given twice daily. These properties of NAOC's give the advantage of shorter hospital stay or managing VTE in an outpatient setting. NOACs should be avoided in severe renal insufficiency (eGFR $<30 \mathrm{ml} / \mathrm{min}$ ) and appropriate dose adjustment and monitoring of renal function at least twice a year be done 
in those with less severe chronic kidney disease ${ }^{16}$. For the patients with cancer associated DVT, LMWH is superior to warfarin ${ }^{17}$ in prevention of VTE and dabiga-tran is as effective as warfarin ${ }^{18}$. Although it has been evident that they are all comparable in efficacy and safety in terms of overall bleeding risks, a retrospective analysis of matched pair cohorts of a large number of participants have shown that in terms of $\mathrm{Gl}$ bleeding apixaban is the safest with lowest risk and rivaroxaban had the highest risk ${ }^{19}$.

\section{Non valvular atrial fibrillation}

Atrial fibrillation $(\mathrm{AF})$ is the most common cardiac arrhythmia in clinical practice and its prevalence increases with age. Approximately $75 \%$ of patients with AF are above the age of 65 and is associated with significant mortality and morbidity as a result of thromboembolism. Warfarin is traditionally used for prevention of stroke and is currently the only approved oral anticoagulant for patients with valvular AF (mitral stenosis or prosthetic heart valves).

NOAC's are a suitable alternative to warfarin and is being increasingly used for prevention of stroke or systemic embolism in patients with non valvular AF. It is at least as effective if not better than warfarin. Currently it is not recommended when AF is associated with mitral or mechanical valves. Apixaban $5 \mathrm{mg}$ twice daily is superior to warfarin in reduction of incidence of stroke (particularly haemorrhagic stroke), major bleeding and all-cause mortality ${ }^{20}$. Dabigatran $150 \mathrm{mg}$ twice daily is more effective than warfarin in overall reduction of incidence of stroke, intracranial haemorrhage $^{21}$ ), and hemorrhagic stroke ${ }^{22}$. Rivaroxaban 15 $\mathrm{mg}$ twice daily is as effective as warfarin in reducing the risk of stroke or systemic embolism with similar incidence of major bleeding ${ }^{23}$. Edoxaban is the newest NAOC to be approved is similar in efficacy to warfarin in reducing overall stroke and risk of overall bleeding ${ }^{24}$. With the given evidence showing that all NAOC's prevent strokes at least as effectively as warfarin with less intracranial bleeding and death the 2016 European Society of Cardiology (ESC) Guidelines now recommends NAOCs as first line anticoagulants for non valvular AF in eligible patients.

\section{Choice of NOAC'S}

All NOAC's are indicated in the management of VTE or NVAF. However, the choice of NAOC's may be influenced by several factors. In patients with high risk of coronary artery disease or previous myocardial infarction rivaroxaban is more preferable ${ }^{23}$. In patients

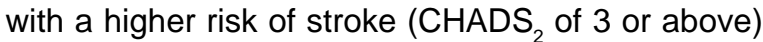
dabigatran gives the greatest stroke reduction ${ }^{25}$ followed by rivaroxaban as the next best choice ${ }^{23}$.
Apixaban causes less GI bleeding than other NAOC's and is the preferred NAOC in those with high risk of $\mathrm{GI}$ bleeding ${ }^{26}$. Rivaroxaban and edoxaban have the convenience of once daily dosing, which is useful to those with poor compliance. Rivaroxaban and apixaban are less dependent on renal elimination and may be safer in those with renal impairment.

\section{Contraindications}

Contraindications for its use include cirrhosis of the liver, pregnancy, breast feeding, children, any conditions associated with major bleeding, presence of additional risk factors for bleeding such as antiplatelet drugs and nonsteroidal anti-inflammatory drugs. NOAC's have variable extent of renal excretion and before prescribing a creatinine clearance should be determined. Dose adjustments should be made if the patient has chronic kidney disease. They are best avoided in severe insufficiency (eGFR $<30 \mathrm{~mL}$ / $\mathrm{min})$. In such patient's warfarin is the anticoagulant of choice. In less severe renal disease (eGFR is $30-50 \mathrm{~mL} / \mathrm{min}$ ) NOAC's are at least as safe as warfarin in terms of bleeding risk $^{27,28}$. Patients with chronic kidney disease who are started on NAOC's should have their renal function monitored every 3 months and those with normal renal function should monitor every 6 months. Further a liver profile should also be done every 6 months and the drug should be reviewed if there are significant alterations of liver function.

\section{Drug interactions}

When compared to warfarin, drug interactions are not a major problem with NAOC's. However, there are some interactions that could significantly alter their anticoagulant effects. Before starting on NOAC a careful drug history must be obtained and once started all new drugs started thereafter should be checked for interactions. Co prescription of an antiplatelet drug with NOAC's increases the bleeding risk and is best avoided unless there is a compelling indications for their use together. All NAOC's are substrates for P-glycoprotein (P-gp) pathway for metabolism and should be preferably avoided in patients taking P-glycoprotein transporter inhibitors or inducers such as ketoconazole, verapamil, and amiodarone (increasing effect) and rifampicin (decreasing effect) ${ }^{29}$. Rivaroxaban and apixaban are metabolized by cytochrome P 450 3A4. Inducers or inhibitors of this enzymes such as ketoconazole, rifampicin, and clarithromycin are preferably avoided ${ }^{30}$.

\section{Laboratory monitoring}

NOAC's usually do not require routine coagulation monitoring due to their stable pharmacokinetic properties and absence of significant food and drug interactions. However, in emergency situations like major bleeding episodes, taking overdoses or recurrence of thoromboembolism may require coagulation testing. Unlike the INR 
(international normalized ratio) test which is used in monitoring warfarin effect, qualitative tests for NAOC's are not widely available. Further, currently these tests unlike the INR use different reagents in different laboratories making it difficult to accurately quantify its effects $^{31,32}$. Anticoagulant effects of dabigatran can be approximately assessed by prothrombin time (PT) /INR and APTT which are prolonged by its action as thrombin inhibitor. A normal APTT suggest no therapeutic anticoagulant effect of dabigatran. Lack of precise quantitative testing is a drawback when managing major bleeding episodes or treatment failure. The British Committee for Standards in Haematology (BCSH) has made some recommendations for rivaroxaban and apixaban $^{11}$. These guidelines may help the clinicians in situations when laboratory assessment may become necessary.

\section{Recommendations of BCSH}

- Each laboratory should know the sensitivity of its own PT and APTT tests to rivaroxaban and apixaban and advise on interpretation.

- The PT or APTT can be used with most reagents for a crude estimation of the relative intensity of anticoagulation due to rivaroxaban but some patients with therapeutic concentrations will have a normal PT or APTT.

- For rivaroxaban the PT is usually more sensitive than the APTT but cannot be used to determine the drug concentration.

- For apixaban both the PT and APTT are insensitive and patients may have normal coagulation times despite therapeutic concentrations.

- Clotting factor assays performed in the presence of Xa inhibitors should include multiple test plasma dilutions and an assessment of parallelism.

\section{Bleeding complications}

Although NAOC's are increasingly prescribed over warfarin, their bleeding risks still remains a major concern. Meta-analysis of 12 randomized control trials has shown that when compared to warfarin NAOC's causes significant less major bleeding, fatal bleeding, clinically relevant non major bleeding, and total bleeding ${ }^{33}$. Of the NAOC's apixaban and dabigatran has shown significantly lower risk of any form of bleeding, major bleeding and risk of death when compared to warfarin ${ }^{34}$.

Because of the short half-life of NAOC's (7-14 hours) mild bleeding can be managed by stopping the drug alone for a brief period. When major bleeding occurs standard supportive measures are the mainstay of treatment. Oral activated charcoal is effective in reducing the rate of absorption of NOAC's if given within few hours of taking this drug ${ }^{35}$. Endoscopic and surgical methods (pressure dressings, cauterization, suturing), fluid replacement including colloids, blood transfusion, fresh frozen plasma, platelet transfusion (if platelet counts are low) hemodialysis $^{36}$, administration of prothrombin complex concentrate (PCC), or activated prothrombin complex concentrate (aPCC) are all recommended as treatment measures in severe cases $^{37}$. PCC's have 4 factors (II VII IX $X$ ) and currently used to reverse the effects of oral factor Xa inhibitors (apixaban, rivaroxaban, and edoxaban). Other factors that may have predisposed for bleeding such as abnormal renal and liver function, drug interactions should be investigated.

Specific reversal agents for NAOC's are being developed. First agent to be licensed is idarucizumab an antibody fragment administered as a 5 grams' slow iv bolus which specifically binds dabigatran with high affinity has shown to completely reverse the anticoagulant effects within minutes ${ }^{38}$. For oral factor Xa inhibitors a recombinant modified human factor $X a$ protein, andexanet alfa, has been developed as a reversal agent when given iv bolus it reduces anti- factor Xa activity within 2-5 minutes ${ }^{39}$. This drug is awaiting licensing late this year.

\section{Patients undergoing interventions while taking NAOC's}

In patients undergoing minor intervention like tooth extraction, cataract surgery, joint injection and skin surgery, NAOC's can be continued provided peak concentrations are avoided and the procedure performed 12 to 24 hours after the last dose. This is an advantage due to their short half-life. However, in situations where significant bleeding is anticipated the drug should be preferably stopped for 1-2 days before the procedure and restarted $24-48$ hours after.

\section{Switching between anticoagulants}

When changing from warfarin to NAOC, it's important to ensure adequate anticoagulation is maintained with minimal bleeding risk. After warfarin is discontinued, rivaroxaban should be started when the INR is less than 3 while apixaban and dabigatran should be started when INR is less than 2. On the other hand, if warfarin is substituted for NOAC, warfarin is started concurrently with standard initial doses and the NAOC is stopped after achieving an INR between 2-3.

\section{Other disadvantages}

Cost of the drugs is a major drawback. Nevertheless, when considering the indirect total costs of warfarin such as regular INR monitoring, clinic visits, management of bleeding complications which are much higher than NAOC's the difference could be very minimal. 
Until now non availability of specific antidote has been a problem in situations like bleeding due to overdose or traumatic injury. Unlike warfarin the experience of using NAOC's is limited to few years only and the long term safety is therefore not known. Additionally, its use is not recommended in those with severe renal and hepatic disease, mechanical or rheumatic heart valves, very young (less than 18 years), pregnancy and elderly.

\section{Conclusion}

Warfarin is a time tested anticoagulant that has the advantages of measurable anticoagulant activity, wide availability and safety in those with chronic kidney disease and other wider range of illnesses. It is the only anticoagulant currently approved for valvular AF.

Patient's adherence to long term warfarin treatment is a problem because most patients with AF are older and treatment compliance and regular clinic visits for monitoring can be difficult. This becomes more difficult if they have to travel long distance to clinics complicated by transport problems that exists in less developed areas in Sri Lanka. The advantages of NAOC's over warfarin has made them the gold standard, for use in NVAF and VTE. The issues such as higher cost, absence of readily available antidotes could be overcome in the near future by reducing the cost of these drugs and improving the availability of the drug and its antidotes.

The most recent (2016) ESC guidelines recommends vitamin $\mathrm{K}$ antagonist therapy (INR 2.0-3.0 or higher) for stroke prevention in AF patients with moderate-to-severe mitral stenosis or mechanical heart valves. However, for those with non valvular AF a NOAC's (apixaban, dabigatran, edoxaban, or rivaroxaban) are recommended in preference to a Vitamin $\mathrm{K}$ antagonist. If non valvular AF patients are already on treatment with a vitamin $\mathrm{K}$ antagonist, they may be considered for NOAC treatment if they are not effectively maintained in therapeutic range of INR or having any practical difficulties in using them.

\section{References}

1. Ageno W, Gallus AS, Wittkowsky A, et al. Antithrombotic therapy and prevention of thrombosis, 9th ed: American College of Chest Physicians evidence-based clinical practice guidelines. Chest 2012; 141 (2 suppl): e44S-e88S

2. Oake N, Fergusson DA, Forster AJ, van Walraven C. Frequency of adverse events in patients with poor anticoa-gulation: a meta-analysis. CMAJ 2007; 176: 1589-94.
3. Deitelzweig S, Amin A, Yonghua J, et al. Comparison of total medical cost avoidance with the usage of new oral anticoagulants instead of warfarin among atrial fibrillation patients, based on the Aristotle, RE-LY and Rocket-AF trials. J Am Coll Cardiol 2012; 59(13): E599.

4. Weitz Jl. Factor Xa or thrombin: is thrombin a better target. J Thromb Haemost 2007; 5(Suppl 1): 65-7.

5. Yong CM, Boyle AJ. Factor Xa inhibitors in acute coronary syndromes and venous thromboembolism. Curr Vasc Pharmacol 2010; 8: 5-11.

6. Schulman S, Kakkar AK, Goldhaber SZ, et al. RE-COVER II Trial Investigators. Treatment of acute venous thromboembolism with dabigatran or warfarin and pooled analysis. Circulation 2014; 129: 764-72.

7. Prins $\mathrm{MH}$, Lensing $\mathrm{AW}$, Bauersachs $\mathrm{R}$, et al. Oral rivaroxaban versus standard therapy for the treatment of symptomatic venous thromboembolism: a pooled analysis of the EINSTEIN-DVT and PE randomized studies. Thromb J 2013; 11: 21.

8. Agnelli G, Buller HR, Cohen A, et al. AMPLIFY Investigators. Oral apixaban for the treatment of acute venous thromboembolism. N Engl J Med 2013; 369: 799-808.

9. Hokusai-VTE Investigators. Edoxaban versus warfarin for the treatment of symptomatic venous thromboembolism. N Engl J Med 2013; 369:1406-15.

10. EINSTEIN-PE Investigators. Oral rivaroxaban for the treatment of symptomatic pulmonary embolism. N Engl J Med 2012; 366: 1287-97.

11. Measurement of non-Coumarin anticoagulants and their effects on tests of Haemostasis: Guidance from the British Committee for Standards in Haematology. British Journal of Haematology 2014; 166: 830-41.

12. National institute for health and clinical excellence. Technology appraisal guidance on Dabigatran etexilate for the treatment and secondary prevention of deep vein thrombosis and/or pulmonary embolism [Internet, last updated 17 November 2014; cited 07 November 2016) Retrieved from https://www.nice.org.uk/Guidance/TA327

13. National institute for health and clinical excellence. Technology appraisal guidance on Edoxaban for treating and for preventing deep vein thrombosis and pulmonary embolism. [Internet, last updated 26 August 2015; cited 07 November 2016) Retrieved from https://www.nice.org. uk/guidance/TA354

14. National institute for health and clinical excellence. Technology appraisal guidance on Rivaroxaban for the treatment of deep vein thrombosis and prevention of recurrent deep vein thrombosis and pulmonary embolism. [Internet, last updated 25 July 2012; cited 07 November 2016) Retrieved from https://www.nice.org.uk/guidance/ ta261

15. National institute for health and clinical excellence. Technology appraisal guidance on Apixaban for the 
treatment and secondary prevention of deep vein thrombosis and/or pulmonary embolism. [Internet, last updated 04 June 2015; cited 07 November 2016) Retrieved from https:// www.nice.org.uk/8guidance/ta341

16. Heidbuchel H, Verhamme P, Alings M, et al. Updated European Heart Rhythm Association practical guide on the use of nonvitamin $\mathrm{K}$ antagonist anticoagulants in patients with nonvalvular atrial fibrillation. Europace 2015; 17: 1467-507.

17. Kearon C, AkI EA, Comerota AJ, et al. Antithrombotic therapy for VTE disease: Antithrombotic Therapy and Prevention of Thrombosis, 9th ed: American College of Chest Physicians Evidence-Based Clinical Practice Guidelines. Chest 2012; 141(2, suppl): e419S-e494S.

18 Schulman S, Kearon C, Kakkar AK, et al. Dabigatran versus warfarin in the treatment of acute venous throm-boembolism. N Engl J Med. 2009; 361(24): 2342-52.

19. Abraham NS et al. Gastrointestinal safety of direct oral anticoagulants: A large population-based study. Gastroenterology 2016 Dec 31; [e-pub]. (http://dx.doi.org/ 10.1053/j.gastro.2016.12.018)

20. Granger $\mathrm{CB}$, Alexander $\mathrm{JH}$, McMurray $\mathrm{JJ}$, et al. ARISTOTLE Committees and Investigators. Apixaban versus warfarin in patients with atrial fibrillation. $N$ Engl J Med. 2011; 365(11): 981-92.

21. Connolly SJ, Ezekowitz MD, Yusuf S, et al. Dabigatran versus warfarin in patients with atrial fibrillation. $N$ Engl J Med. 2009; 361(12): 1139-51.

22. January CT, Wann LS, Alpert JS, et al. 2014 AHA/ACC/ HRS Guideline for the management of patients with atrial fibrillation: a report of the American College of Cardiology/ American Heart Association Task Force on practice guidelines and the Heart Rhythm Society. J Am Coll Cardiol. 2014; 64(21): e1-e76.

23. Patel MR, Mahaffey KW, Garg J, Pan G, Singer DE, Hacke W, et al. Rivaroxaban versus warfarin in nonvalvular atrial fibrillation. N Engl J Med 2011; 365(10): 883-91.

24. Giugliano RP, Ruff CT, Braunwald E, et al. ENGAGE AF-TIMI 48 Investigators. Edoxaban versus warfarin in patients with atrial fibrillation. N Engl J Med. 2013; 369(22): 2093-2104.

25. Oldgren J, Alings M, Darius H, Diener HC, Eikelboom J, Ezekowitz MD, et al. Risks for stroke, bleeding, and death in patients with atrial fibrillation receiving dabigatran or warfarin in relation to the CHADS2 score: a subgroup analysis of the RE-LY trial. Ann Intern Med 2011; 155(10): 660-7, W204.

26. Holster IL, Valkhoff VE, Kuipers EJ, Tjwa ET. New oral anticoagulants increase risk for gastrointestinal bleedinga systematic review and metaanalysis. Gastroenterology 2013; 145(1): 105-12.

27. FoxKA, Piccini JP, Wojdyla D, Becker RC, Halperin JL, Nessel
$\mathrm{CC}$, et al. Prevention of stroke and systemic embolism with rivaroxaban compared with warfarin in patients with nonvalvular atrial fibrillation and moderate renal impairment. Eur Heart J 2011; 32(19): 2387-94.

28. Hohnloser SH, Hijazi Z, Thomas L, Alexander JH, Amerena $\mathrm{J}$, Hanna $\mathrm{H}$, et al. Efficacy of apixaban when compared with warfarin in relation to renal function in patients with atrial fibrillation: insights from the ARISTOTLE trial. Eur Heart J 2012; 33(22): 2821-30.

29. Little JW. New oral anticoagulants: will they replace warfarin? Oral Surg Oral Med Oral Pathol Oral Radiol. 2012; 113(5): 570-80.

30. Walenga JM, Adiguzel C. Drug and dietary interactions of the new and emerging oral anticoagulants. Int J Clin Pract 2010; 64(7): 956-67.

31. Douxfils J, Mullier F, Robert S, Chatelain C, Chatelain B, Dogne JM. Impact of dabigatran on a large panel of routine or specific coagulation assays. Laboratory recommendations for monitoring of dabigatran etexilate. Thromb Haemost 2012; 107: 985-97.

32. Harenberg J, Erdle S, Marx S, Kramer R. Determination of rivaroxaban in human plasma samples. Semin Thromb Hemost 2012; 38: 178-84.

33. Chai-Adisaksopha C, Crowther M, Isayama T, Lim W. The impact of bleeding complications in patients receiving target-specific oral anticoagulants: a systematic review and meta-analysis. Blood 2014; 124: 2450-8.

34. Torben Bjerregaard Larsen, Flemming Skjøth, Peter Brønnum Nielsen, Jette Nordstrøm Kjældgaard, Gregory Y H Lip. Comparative effectiveness and safety of nonvitamin $\mathrm{K}$ antagonist oral anticoagulants and warfarin in patients with atrial fibrillation: propensity weighted nationwide cohort study. BMJ 2016; 353: i3189.

35. Kaatz S, Kouides PA, Garcia DA, et al. Guidance on the emergent reversal of oral thrombin and factor Xa inhibitors. Am J Hematol 2012; 87: S141-S145.

36. Chang DN, Dager WE, Chin Al. Removal of dabigatran by hemodialysis. Am J Kidney Dis. 2013; 61(3): 487-9.

37. van Ryn J, Stangier J, Haertter S, Liesenfeld KH, Wienen W, Feuring $M$ Et al. Dabigatran etexilate a novel, reversible, oral direct thrombin inhibitor: interpretation of coagulation assays and reversal of anticoagulant activity. Thromb Haemost 2010; 103: 1116-27.

38. Pollack CV Jr, Reilly PA, Eikelboom J, Glund S, Verhamme $\mathrm{P}$, Bernstein RA et al.Idarucizumab for dabigatran reversal. N Engl J Med 2015; 373: 511-20.

39. Siegal DM, Curnutte JT, Connolly SJ, et al. Andexanet alfa for the reversal of factor Xa inhibitor activity. $N$ Engl J Med 2015; 373: 2413e24. 